\title{
CHEMICAL BINDING EFFECTS ON RESONANT SCATTERING OF NEUTRONS
}

\author{
A. I. Shamaoun' and G. C. Summerfield ${ }^{2}$ \\ 'Atomic Energy Commission, P.O. Box 6091, Damascus, Syria \\ 'Department of Nuclear Engineering. The University of Michigan. Ann Arbor, M148109-2104. \\ U.S.A.
}

(Received for publication 29 November 1989 )

\begin{abstract}
Earlier calculations of the chemical binding effect on neutron resonance broadening do not agree with experimental results. A possible source of this difference is that the calculations have only examined the effect of chemical binding on resonant absorption and have not investigated the effect on resonant scattering. It is vital that we understand the discrepancy between the computed and measured chemical binding effect. Therefore the resonant differential scattering cross section is calculated for a harmonic crystal model to examine the chemical binding effect for resonant scattering. We show that, at high temperatures or high resonance energies, scattering can be treated using ideal gas with the same effective temperature as for absorption accounting for chemical binding effects. Although the resonance broadening is determined in terms of a four-point correlation function, it is found that this broadening can still be written in terms of the usual $\psi$-function and the effect of chemical binding in both resonance absorption and scattering is not large enough to account for the large measured effect. Thus the experiments that show a substantial amount of chemical binding must be reevaluated.
\end{abstract}

\section{INTRODUCTION}

The broadening of neutron scattering and absorption resonances by the thermal motions of the target atoms is referred to as "Doppler broadening". Doppler broadening is known to depend upon the state of the atom from which the scattering occurs. That is, it depends upon whether the atom is in a liquid, a solid or a gas. This dependence is called the chemical binding effect. It was first discussed by Lamb in (1939). This work led Mössbauer (1958) to his results for resonant absorption of $\gamma$-rays.

When calculating Doppler broadening effects on nuclear reactor parameters, people universally ignore the chemical binding effect and the atom is treated as a gas. However, an effective temperature is sometimes used. There has been a rather considerable amount of theoretical work (Nelkin and Parks, 1960; Karam, 1975: Holger and Karam. 1981; Sher, 1983; Bernabei, 1964) showing that the chemical binding effect can, in fact, be ignored when computing absorption parameters such as Doppler coefficients for nuclear reactors. There are, however, a number of experiments (Tsang and Brugger, 1979, 1980; Fujita et al., 1984) which do show a substantial chemical binding effect for neutron resonances even at neutron energies in the $\mathrm{keV}$ energy range. In their experiments, the effective neutron resonance cross section is as much as $23 \%$ different between $\mathrm{U}$ metal and $\mathrm{U}$ oxide for $24 \mathrm{keV}$ neutrons. Similar large differences have been observed between metal and carbide and solid and molten metal. Such effects would be of considerable importance when computing the parameters for a fast reactor. It is vital that we understand the discrepancy between the computed and measured chemical binding effect and determine if the measured effect is real.

Previous calculations have only examined the effect of chemical binding on absorption and have not investigated the effect on resonant scattering. We have examined the underlying approximations in the resonance absorption forms used in the computations applied to an atom harmonically bound in a crystal (Shamaoun, 1989). We found the same lack of a large chemical binding effect. In particular, one of the approximations made in computing the chemical binding effect is that the neutron and $\gamma$ line widths do not depend upon the chemical binding state of the compound nucleus. This is an excellent approximation for the $\gamma$ line width. We have calculated the effects of the dependence of the neutron line width upon the atomic state of the compound nucleus (Shamaoun and Summerfield, 1989). While the effects are there, they are not large enough to explain the experimental observations. 
Djafri (1988) has used an anharmonic model to compute the broadening of the absorption cross section. His results, which show a large effect particularly in a high temperature limit, are of doubtful validity. He uses an approximation to represent the "phonon damping" to the correlation function which is only valid at large times. He gets a large effect in the wings of the resonance which is mostly effected by the correlation function at small times. We should also note that Djafri's expressions do not go to the correct high temperature limit which is :

$$
\left\langle\exp \left[-i \mathbf{K} \cdot \mathbf{R}_{L}(t)\right] \exp \left(i \mathbf{K} \cdot \mathbf{R}_{L}\right)\right\rangle_{T}=\exp \left\{-\frac{i \hbar K^{2}}{2 M_{L}} t-\frac{K^{2}}{2 M_{L}}\left\langle\frac{P_{L}^{2}}{2 M_{L}}\right\rangle_{T} t^{2}+\cdots\right\},
$$

where $h \mathbf{K}$ is the momentum of the neutron and $\mathbf{P}_{L}$ is the momentum of the $L$ th atom in the crystal, whose coordinates are $\mathbf{R}_{L}$. Therefore his "phonon damping" method is not a good way to include anharmonic contributions in resonant neutron absorption.

\section{GENERAL FORMULATION}

The cross-section for the capture of a slow neutron with an energy near to a resonance level of a nucleus which accounts for the thermal motion in an ideal gas is given by Bethe and Placzek (1937) interms of the $\psi$ function:

$$
\sigma_{\mathrm{n}, \gamma}=\sigma_{\mathrm{n}, \gamma_{0}} \psi(x, \xi)
$$

where $\psi(x, \xi)$ is called the Doppler integral (Westinghouse Atomic Power Division, 1954) and is given by

$$
\psi(x, \xi)=\frac{\xi}{2 \sqrt{\pi}} \int_{-\infty}^{\infty} \mathrm{d} y \frac{\exp \left[-\frac{\xi^{2}}{4}(x-y)^{2}\right]}{1+y^{2}},
$$

where:

$$
\begin{gathered}
\xi=\frac{\Gamma}{\Delta}, \\
x=\frac{2}{\Gamma}\left(E_{\mathrm{n}}-E_{0}-R\right) .
\end{gathered}
$$

The Doppler width $\Delta$ is given by $\Delta=2(R / \beta)^{1 / 2}$ where $\beta^{-1}$ is the temperature (multiplied by Boltzmann constant $k_{\mathrm{B}}$ ) in energy units. Such reactions proceed via a compound nucleus which decays by emitting a highenergy $\gamma$.

The other process for the interaction of slow neutrons is elastic scattering. This interaction also proceeds via a compound nucleus which decays by the reemission of the neutron with the target nucleus returning to its ground state. The elastic cross section has been written as a sum of three terms for an ideal gas:

$$
\sigma_{\mathrm{n}, \mathrm{n}}=\sigma_{\mathrm{n}, \mathrm{n}_{0}} \psi(x, \xi)+\sigma_{\mathrm{n}, \mathrm{n}_{\mathrm{o}}} \frac{\Gamma}{\Gamma_{\mathrm{n}}} \frac{2 R}{\lambda_{0}} \Phi(x, \xi)+4 \pi R^{2},
$$

where $R$ is the nuclear radius (given approximately by $R \sim 1.25 \times 10^{-13} \mathrm{~A}^{1 / 3} \mathrm{~cm}$ ), $\lambda_{0}$ is the reduced neutron wavelength and $\Phi(x, \xi)$ is another integral (Hass and Shore, 1959) given by:

$$
\Phi(x, \xi)=\frac{\xi}{2 \sqrt{\pi}} \int_{-\infty}^{\infty} y \frac{\exp \left[-\frac{\xi^{2}}{4}(x-y)^{2}\right]}{1+y^{2}} \mathrm{~d} y .
$$

The first term of equation (6) represents the elastic scattering cross section due to the reemission of the neutron and the second term is the interference scattering. The simplest type of nuclear interaction is the last term called potential scattering in which the incident neutron scatters elastically off of the nuclear potential without penetrating the nuclear surface. Such 'billiard-ball' collisions are characterized by an essentially energy 
independent cross section $\sigma_{\mathrm{p}}$. The total broadened resonance cross section $\sigma_{\mathrm{n} \cdot \mathrm{T}}$ is given by the sum of the capture and scattering term:

$$
\sigma_{\mathrm{n}, \mathrm{T}}=\left[\sigma_{\mathrm{n}, \gamma_{0}}+\sigma_{\mathrm{n}, \mathrm{n}_{\mathrm{n}}}\right] \psi(x, \xi)+\sigma_{\mathrm{n}, \mathrm{n}_{\mathrm{n}}} \frac{\Gamma}{\Gamma_{\mathrm{n}}} \frac{2 R}{\lambda_{0}} \Phi(x, \xi)+4 \pi R^{2} .
$$

At high neutron energies, the atom recoil is so large so that the chemical binding will have no influence on such cross sections due to the extremely large energies involved compared to the atoms' binding energies.

\section{RESONANT SCATTERING CROSS SECTION}

The starting point of the development is the usual formula of the differential scattering cross section for the scattering of thermal neutrons (Lovesey and Springer, 1977):

$$
\frac{\mathrm{d}^{2} \sigma}{\mathrm{d} \epsilon \mathrm{d} \Omega}=\sum_{\mathrm{i}, \mathrm{f}}\left(\frac{E_{\mathrm{f}}}{E_{\mathrm{i}}}\right)^{1 / 2} g\left(\varepsilon_{\mathrm{i}}\right)\left|\frac{m}{2 \pi h^{2}}\langle f|T| i\rangle\right|^{2} \delta\left(E_{\mathrm{i}}+\varepsilon_{\mathrm{i}}-E_{\mathrm{f}}-\varepsilon_{\mathrm{f}}\right),
$$

where $\boldsymbol{t}$ is the energy transfered to the neutron upon scattering:

$$
\epsilon=\frac{\hbar^{2}\left(K_{\mathrm{f}}^{2}-K_{\mathrm{i}}^{2}\right)}{2 m}=E_{\mathrm{r}}-E_{\mathrm{i}}
$$

$E_{\mathrm{i}}$ and $E_{\mathrm{f}}$ are the initial and final neutron energies respectively, $\varepsilon_{\mathrm{i}}$ and $\varepsilon_{\mathrm{f}}$ are the target energies and $m$ is the neutron mass.

A derivation of the differential cross section has been given by Goodman and Waller in (1966). Equation (9) represents the most generally valid formula for the resonance cross section per unit solid angle and unit energy gain averaged over all possible initial and final target system states weighted by the statistical probabilities $g\left(c_{i}\right)$ for the initial state. The $\delta$-function represents the conservation of energy. The matrix $\langle f|T| i\rangle$ for resonant scattering is (Shamaoun and Summerfield, 1989):

$$
\langle f|T| i\rangle=\langle f|V| j\rangle+\sum_{j} \frac{\langle f|V| j\rangle\langle j|V| i\rangle}{E-E_{0}+\frac{i \Gamma(j)}{2}}+\cdots
$$

where

$$
E_{0}=E_{j}+\langle j|V| j\rangle+P \sum_{i \neq j} \frac{\langle j|V| l\rangle\langle l|V| j\rangle}{E-E_{l}}+\cdots .
$$

The second and third terms on the right-hand-side of equation (12) are shifts in the resonance energy which. in principle, depend upon the atomic state of the compound nucleus. The energy of the system in the intermediate state $E_{j}$ is just the resonance energy plus the eigenenergy of the Hamiltonian $\mathrm{H}_{\mathrm{A}}$. The second term, $\langle j|V| j\rangle$, is zero for our case. This is true since $V$ must connect the compound nucleus state with a state in which there is either an emitted photon or neutron. The diagonal matrix elements of $V$ must vanish. We ignore the third term. The total energy of the system in the initial state is:

$$
E=\frac{\hbar^{2} K_{i}^{2}}{2 m}+\varepsilon_{i}=E_{i}+\varepsilon_{1}
$$

The square of the absolute value of the $T$-matrix in equation (11) consists of three terms. The square of the first term is the potential part and the cross product term is the interference part and the square of the second term is the resonance part. Here, we only examine the resonance part. The matrix elements $\langle f|V| j\rangle$ and $\langle j|V| i\rangle$ are given by (Shamaoun and Summerfield, 1989):

$$
\begin{aligned}
\langle f|V| j\rangle & =\left|M_{\text {comp }}\right|\left\langle f\left|\exp \left(-i \mathbf{K}_{\mathrm{f}} \cdot \mathbf{R}_{L}\right)\right| j\right\rangle, \\
\langle j|V| i\rangle & =\left|M_{\text {comp }}^{\prime}\right|\left\langle j\left|\exp \left(i \mathbf{K}_{\mathrm{i}} \cdot \mathbf{R}_{L}\right)\right| i\right\rangle,
\end{aligned}
$$

where $\mathbf{K}_{1}$ and $\mathbf{K}_{\mathrm{f}}$ are the neutron's initial and final wave vectors, $\mathbf{R}_{\ell}$ specifies the position of the $L$ th scattering 
nucleus in the laboratory system and $\left|M_{\text {comp }}\right|$ and $\left|M_{\text {comp }}^{\prime}\right|$ are the purely nuclear matrix elements described by the following:

$$
\begin{aligned}
\left|M_{\text {comp }}\right| & =\int \mathrm{d} r^{3} \exp \left(i \mathbf{K}_{\mathrm{f}} \cdot \mathbf{r}\right) V(r) \Psi^{*}(r) \\
\left|M_{\text {comp }}^{\prime}\right| & =\int \mathrm{d} r^{3} \exp \left(-i \mathbf{K}_{\mathrm{i}} \cdot \mathbf{r}\right) V(r)\left[\Psi^{*}(r)\right]^{*}
\end{aligned}
$$

$\Psi^{*}(r)$ is the excited state of the compound nucleus as a function of relative coordinate $r$ and $\left[\Psi^{*}(r)\right]^{*}$ its conjugate. Therefore the resonance part of equation (11) can be written as :

$$
\langle f|T|\rangle=\left|M_{\text {comp }}\right|\left|M_{\text {comp }}^{\prime}\right| \sum_{j} \frac{\left\langle f\left|\exp \left(-i \mathbf{K}_{\mathrm{f}} \cdot \mathbf{R}_{L}\right)\right| j\right\rangle\left\langle j\left|\exp \left(i \mathbf{K}_{i} \cdot \mathbf{R}_{L}\right)\right| i\right\rangle}{E_{\mathrm{i}}-E_{0}+\varepsilon_{\mathrm{i}}-\varepsilon_{j}+\frac{i \Gamma}{2}} .
$$

This equation is very similar to the transition probability of Osborn and Yip (1966). It was first shown by Van Hove in (1954) that equation (9) may be expressed in terms of microscopic two-time correlation functions for non-resonance scattering. Trammell (1966) and later Word and Trammell (1981) generalized this work to give an expression for the resonant scattering from nuclei in condensed matter. Their expression contains a fourpoint correlation function. Below we repear derivation of the expression of the resonant scattering broadening in terms of the four-point correlation function.

\section{DERIVATION OF RESONANT CORRELATION FUNCTION FORMALISM}

We can transform the denominator of the expression (18) into a time-dependent representation (Van Hove, 1954 ; Singwi and Sjolander, 1960):

$$
\frac{1}{E_{\mathrm{f}}-E_{0}+\varepsilon_{\mathrm{f}}-\varepsilon_{j}+\frac{i \Gamma}{2}}=-i \int_{0}^{\infty} \frac{\mathrm{d} t}{\hbar} \exp \left[-i\left(E_{\mathrm{f}}-E_{0}+\varepsilon_{\mathrm{f}}-\varepsilon_{j}+\frac{i \Gamma}{2}\right) \frac{t}{\hbar}\right],
$$

where we have replaced $E_{\mathrm{i}}+\varepsilon_{\mathrm{i}}$ by $E_{\mathrm{f}}+\varepsilon_{\mathrm{f}}$ using the conservation of energy. Also, we assume that $\Gamma$ is independent of the state $j$ of the compound nucleus. Then equation (18) becomes:

$$
\begin{aligned}
\langle f|T| i\rangle=\left|M_{\text {comp }}\right|\left|M_{\text {comp }}^{\prime}\right| \sum_{j}-i \int_{0}^{\infty} \frac{\mathrm{d} t}{\hbar} \exp \left[-i\left(\Delta E+\varepsilon_{\mathrm{f}}-\varepsilon_{j}+\frac{i \Gamma}{2}\right) \frac{t}{h}\right] \\
\times(x)\left\langle f\left|\exp \left(-i \mathbf{K}_{\mathrm{f}} \cdot \mathbf{R}_{L}\right)\right| j\right\rangle\left\langle j\left|\exp \left(i \mathbf{K}_{\mathrm{i}} \cdot \mathbf{R}_{L}\right)\right| i\right\rangle,
\end{aligned}
$$

substituted into (9)

$$
\begin{aligned}
\frac{\mathrm{d}^{2} \sigma}{\mathrm{d} \epsilon \mathrm{d} \Omega}=Q \sum_{\mathrm{i}, \mathrm{f}}\left(\frac{E_{\mathrm{f}}}{E_{\mathrm{i}}}\right)^{1 / 2} g\left(\varepsilon_{\mathrm{i}}\right) \mid \sum_{j}-i \int_{0}^{\infty} \frac{\mathrm{d} t}{\hbar} \exp [ & -i\left(\Delta E+\varepsilon_{\mathrm{f}}-\varepsilon_{j}+\frac{i \Gamma}{2}\right) \frac{t}{\hbar} \\
& \times(x)\left\langle f\left|\exp \left(-i \mathbf{K}_{\mathrm{f}} \cdot \mathbf{R}_{L}\right)\right| j\right\rangle\left\langle j\left|\exp \left(i \mathbf{K}_{\mathrm{i}} \cdot{ }_{i}\right)\right| i\right\rangle \mid \delta\left(-\epsilon+\varepsilon_{\mathrm{i}}-\varepsilon_{\mathrm{f}}\right),
\end{aligned}
$$

where $\Delta E=E_{\mathrm{f}}-E_{0}$ and $Q$ represents the nuclear factor :

$$
Q=\left(\frac{m}{2 \pi \hbar^{2}}\right)^{2}\left|M_{\text {comp }}\right|^{2}\left|M_{\text {comp }}^{\prime}\right|^{2},
$$

using

$$
\exp \left(i \varepsilon_{\mathrm{f}} \frac{t}{h}\right)|f\rangle=\exp \left(i \mathrm{H}_{\mathrm{A}} \frac{t}{h}\right)|f\rangle
$$




$$
\begin{gathered}
\exp \left(-i \varepsilon_{j} \frac{t}{h}\right)|j\rangle=\exp \left(-i \mathrm{H}_{\mathrm{A}} \frac{l}{h}\right)|j\rangle . \\
\sum_{j}|j\rangle\langle j|=I,
\end{gathered}
$$

we can writc

$$
\begin{aligned}
\sum_{i}\langle f| \exp \left(i \mathrm{H}_{\mathrm{A}} \frac{t}{\hbar}\right) & \exp \left(-i \mathbf{K}_{\mathrm{r}} \cdot \mathbf{R}_{L}\right)|j\rangle\left\langle j\left|\exp \left(-i \mathrm{H}_{\mathrm{A}} \frac{t}{\hbar}\right) \exp \left(i \mathbf{K}_{\mathrm{i}} \cdot \mathbf{R}_{I}\right)\right| i\right\rangle \\
& =\left\langle f\left|\exp \left[-\mathrm{i} \mathbf{K}_{\mathrm{r}} \cdot \mathbf{R}_{L}(t)\right] \exp \left(i \mathbf{K}_{\mathrm{i}} \cdot \mathbf{R}_{I}\right)\right| i\right\rangle,
\end{aligned}
$$

also using

$$
\begin{gathered}
\delta(x)=\frac{1}{2 \pi} \int_{-,}^{\star x} \operatorname{d} T \\
\exp \left(i \varepsilon_{\mathrm{i}} \frac{T}{\hbar}\right)|i\rangle=\exp \left(i \mathrm{H}_{\mathrm{A}} \frac{T}{\hbar}\right)|i\rangle, \\
\exp \left(-i \varepsilon_{\mathrm{f}} \frac{T}{\hbar}\right)|f\rangle=\exp \left(-i \mathrm{H}_{\mathrm{A}} \frac{T}{\hbar}\right)|f\rangle, \\
\sum|f\rangle\langle f|=I,
\end{gathered}
$$

the square of the above matrix element can be written as thermal average of a four-point correlation function :

$$
\begin{aligned}
\sum_{i, \mathrm{f}} g\left(c_{\mathrm{i}}\right)\left|\left\langle f\left|\exp \left[-i \mathbf{K}_{\mathrm{r}} \cdot \mathbf{R}_{L}(t)\right] \exp \left(i \mathbf{K}_{\mathrm{i}} \cdot \mathbf{R}_{L}\right)\right| i\right\rangle\right|^{2} \\
=\left\langle\exp \left[-i \mathbf{K}_{\mathrm{i}} \cdot \mathbf{R}_{I .}(T)\right] \exp \left[i \mathbf{K}_{\mathrm{f}} \cdot \mathbf{R}_{L,}\left(T+t^{\prime}\right)\right] \exp \left[-i \mathbf{K}_{\mathrm{f}} \cdot \mathbf{R}_{L}(t)\right] \exp \left(i \mathbf{K}_{\mathrm{i}} \cdot \mathbf{R}_{l}\right)\right\rangle_{T} .
\end{aligned}
$$

Therefore equation (21) becomes:

$$
\begin{aligned}
& \frac{\mathrm{d}^{2} \sigma}{\mathrm{d} \epsilon \mathrm{d} \Omega}=Q\left(\frac{E_{r}}{E_{\mathrm{i}}}\right)^{1 / 2} \int^{\prime} \frac{\mathrm{d} T}{h_{1}} \exp \left(-i \varepsilon \frac{T}{h}\right) \int_{0}^{\infty} \frac{\mathrm{d} t}{\hbar} \exp \left[i\left(\Delta E+\frac{i \Gamma}{2}\right) \frac{t}{h}\right] \int_{0}^{\infty} \frac{\mathrm{d} t^{\prime}}{h} \exp \left[-i\left(\Delta E+\frac{i \Gamma}{2}\right) \frac{t^{\prime}}{h}\right] \\
& \times(x)\left\langle\exp \left[-i \mathbf{K}_{\mathrm{i}} \cdot \mathbf{R}_{L}(T)\right] \exp \left[i \mathbf{K}_{\mathrm{f}} \cdot \mathbf{R}_{I}\left(T+t^{\prime}\right)\right] \exp \left[\left(-i \mathbf{K}_{\mathrm{f}} \cdot \mathbf{R}_{, L}(t)\right] \exp \left(i \mathbf{K}_{\mathrm{i}} \cdot \mathbf{R}_{,}\right)\right\rangle_{r} .\right.
\end{aligned}
$$

This equation represents the basic result for resonant neutron incoherent scattering from the $L$ th atom. It shows that the resonant scattering broadening is determined by a four-time correlation function. This broadening seems to be quite different from the resonant absorption broadening which is determined by the two-point correlation. The effect of chemical binding on resonant scattering has never been computed in detail for the harmonic crystal. Therefore, the purpose of this paper is to compute the effects of the chemical on the resonant scattering cross section at lower energies.

\section{ELASTIC SCATTERING OF NEUTRONS BY HARMONIC CRYSTALS}

In here we consider neutron scattering from a harmonic crystal. In this model, the atoms are assumed to undergo oscillations about equilibrium positions:

$$
\mathbf{r}_{i l .}=\boldsymbol{\rho}_{j}+\mathbf{r}_{L}
$$

where the $\rho_{j}$ is the position of the $j$ th unit cell, and $\mathbf{r}_{L}$ the equilibrium position of the $L$ th atom is the unit cells form a simple Bravais lattice described by:

$$
\boldsymbol{\rho}_{i}=j_{1} \mathbf{d}_{1}+j_{2} \mathbf{d}_{2}+j_{3} \mathbf{d}_{3},
$$


where $j_{1}, j_{2}, j_{3}=0, \pm 1, \pm 2, \ldots$ and $\mathbf{d}_{i}$ are constant, called basis vectors of the lattice. The position of the $L$ th atom in the $j$ th cell at any time is :

$$
\mathbf{R}_{j L}(t)=\mathbf{r}_{j L}+\mu_{j L}(t),
$$

where $\boldsymbol{\mu}_{j L}(t)$ is the displacement of the $L$ th atom in the unit cell $j$ from its equilibrium value $\mathbf{r}_{j L}$. Complete discussions of the quantum mechanical description of the harmonic crystal are readily available in any number of references (Weinreich, 1965; Born and Huang, 1954).

In order to evaluate the incoherent resonance cross section [equation (32)] for the case of a harmonic model of a crystal lattice, we first evaluate the thermal average:

$$
\left\langle\exp \left[i \mathbf{K}_{\mathrm{i}} \cdot \mathbf{R}_{L}(T)\right] \exp \left[i \mathbf{K}_{\mathrm{f}} \cdot \mathbf{R}_{L}\left(T+t^{\prime}\right)\right] \exp \left[-i \mathbf{K}_{\mathrm{f}} \cdot \mathbf{R}_{L}(t)\right] \exp \left(i \mathbf{K}_{\mathrm{i}} \cdot \mathbf{R}_{L}\right)\right\rangle_{T} .
$$

It is possible to evaluate these expressions directly for a harmonically oscillating system by starting from the usual mode analysis of the crystal vibration (Born and Huang, 1954):

$$
\boldsymbol{\mu}_{j L}(t)=\sum_{\mathbf{q} \lambda}\left(\frac{\hbar}{2 N M_{L} w_{\lambda}}\right)^{1 / 2}\left\{\mathbf{e}_{\mathbf{q} \lambda}^{L} a_{\mathbf{q} \lambda} \exp \left(i \mathbf{q} \cdot \boldsymbol{\rho}_{j}-i w_{\lambda} t\right)+\mathbf{e}_{\mathbf{q} \lambda}^{L^{*}} a_{\mathbf{q} \lambda}^{+} \exp \left(-i \mathbf{q} \cdot \boldsymbol{\rho}_{j}+i w_{\lambda} t\right)\right\},
$$

where $a_{\mathrm{q} \lambda}$ and $a_{\mathrm{q} \lambda}^{+}$are the creation and annihilation operators, $\mathbf{e}_{\mathbf{q} \lambda}^{L}$ is the polarization factor ( $\lambda$ is the vibration branch for the wave vector $\mathbf{q}), w_{\lambda}(\mathbf{q})$ is the normal mode frequency, $N$ is the number of unit cells, and $M_{L}$ is the $L$ th atomic mass. The polarization vectors obey the orthogonality relation:

$$
\sum_{L} \mathbf{e}_{\mathbf{q} \lambda}^{L^{*}} \mathbf{e}_{\mathbf{q} \lambda^{\prime}}^{L}=\delta_{i \lambda}
$$

We use Weyl's identity (1927) to combine the exponents in (36):

$$
\exp (A) \exp (B)=\exp \left(A+B+\frac{1}{2}[A, B]\right),
$$

where double and higher commutators are zero for harmonic oscillator coordinates:

$\exp (A) \exp (B) \exp (C) \exp (D)$

$$
=\exp \left\{A+B+C+D+\frac{1}{2}[A, B]+\frac{1}{2}[A, C]+\frac{1}{2}[A, D]+\frac{1}{2}[B, C]+\frac{1}{2}[B, D]+\frac{1}{2}[C, D]\right\} .
$$

Furthermore, we use Bloch's (1932) theorem:

$$
\langle\exp (Q)\rangle_{T}=\exp \left(\frac{1}{2}\left\langle Q^{2}\right\rangle_{T}\right.
$$

Then the thermal averages in (36) can be written :

$$
\begin{aligned}
\langle\exp [ & \left.-i \mathbf{K}_{\mathrm{i}} \cdot \mathbf{R}_{L}(T)\right] \exp \left[i \mathbf{K}_{\mathrm{f}} \cdot \mathbf{R}_{L}\left(T+t^{\prime}\right)\right] \exp \left[-i \mathbf{K}_{\mathrm{f}} \cdot \mathbf{R}_{L}(t)\right] \exp \left(i \mathbf{K}_{\mathrm{i}} \cdot \mathbf{R}_{L}\right\rangle_{T} \\
= & \exp \left\{-\frac{1}{2}\left[\mathbf{K}_{\mathrm{f}} \cdot \mathbf{R}_{L}\left(T+t^{\prime}\right), \mathbf{K}_{\mathrm{i}} \cdot \mathbf{R}_{L}\right]-\frac{1}{2}\left[-\mathbf{K}_{\mathrm{f}} \cdot \mathbf{R}_{L}(t), \mathbf{K}_{\mathrm{i}} \cdot \mathbf{R}_{\iota}\right]-\frac{1}{2}\left[-\mathbf{K}_{\mathrm{i}} \cdot \mathbf{R}_{L}(T), \mathbf{K}_{\mathrm{i}} \cdot \mathbf{R}_{L}\right]\right. \\
& -\frac{1}{2}\left[\mathbf{K}_{\mathrm{f}} \cdot \mathbf{R}_{L}\left(T+t^{\prime}\right),-\mathbf{K}_{\mathrm{f}} \cdot \mathbf{R}_{L}(t)\right]-\frac{1}{2}\left[-\mathbf{K}_{\mathrm{i}} \cdot \mathbf{R}_{L}(T), \mathbf{K}_{\mathrm{f}} \cdot \mathbf{R}_{L}\left(T+t^{\prime}\right)\right]-\frac{1}{2}\left[\mathbf{K}_{\mathrm{i}} \cdot \mathbf{R}_{L}(T), \mathbf{K}_{\mathrm{f}} \cdot \mathbf{R}_{L}(t)\right] \\
& \left.-\frac{1}{2}\left\langle\left[-\mathbf{K}_{\mathrm{i}} \cdot \mathbf{R}_{L}(T)+\mathbf{K}_{\mathrm{f}} \cdot \mathbf{R}_{L}\left(T+t^{\prime}\right)-\mathbf{K}_{\mathrm{f}} \cdot \mathbf{R}_{L}(t)+\mathbf{K}_{\mathrm{i}} \cdot \mathbf{R}_{L}\right]^{2}\right\rangle_{T}\right\} .
\end{aligned}
$$

Each of the commutator terms can be calculated using equation (35) and the following commutation relation:

$$
\begin{aligned}
& {\left[a_{\mathbf{q} \lambda}, a_{\mathbf{q}^{\prime} \lambda^{\prime}}^{+}\right]=\delta_{\lambda \lambda^{\prime}} \delta\left(\mathbf{q}^{\prime}-\mathbf{q}\right),} \\
& {\left[a_{\mathbf{q} \lambda}, a_{\mathbf{q}^{\prime} \lambda^{\prime}}\right]=\left[a_{\mathbf{q}^{\prime} \lambda}^{+}, a_{\mathbf{q}^{\prime} \lambda^{\prime}}^{+}\right]=0 .}
\end{aligned}
$$

We obtain

$$
\begin{gathered}
{\left[\mathbf{K}_{\mathrm{f}} \cdot \mathbf{R}_{L}\left(T+t^{\prime}\right), \mathbf{K}_{\mathrm{i}} \cdot \mathbf{R}_{L}\right]=\sum_{\mathbf{q} \lambda} \frac{\hbar}{2 N M_{L} W_{i}},} \\
\left\{\left(\mathbf{K}_{\mathrm{i}} \cdot \mathbf{e}_{\mathbf{q} \lambda}^{L}\right)\left(\mathbf{K}_{\mathrm{i}} \cdot \mathbf{e}_{\mathbf{q} \lambda}^{l^{*}}\right) \exp \left[-i w_{\lambda}\left(T+t^{\prime}\right)\right]-\left(\mathbf{K}_{\mathbf{i}} \cdot \mathbf{e}_{\mathbf{q} \lambda}^{L^{*}}\right)\left(\mathbf{K}_{\mathbf{f}} \cdot \mathbf{e}_{\mathbf{q} \lambda}^{L}\right) \exp \left[i w_{\lambda}\left(T+t^{\prime}\right)\right]\right\},
\end{gathered}
$$




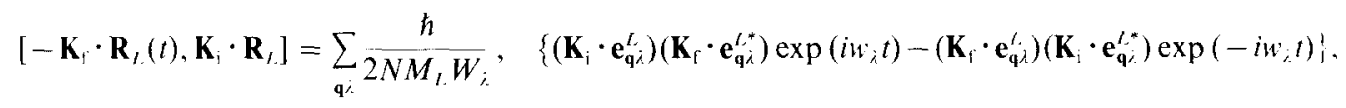

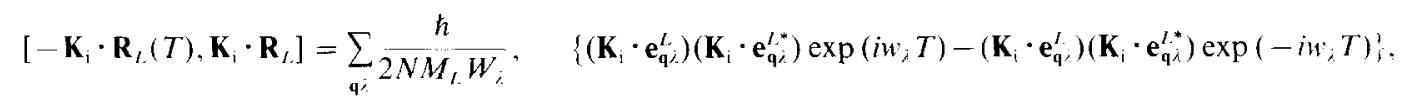

$\left[\mathbf{K}_{\mathrm{f}} \cdot \mathbf{R}_{\iota}\left(T+t^{\prime}\right),-\mathbf{K}_{\mathrm{f}} \cdot \mathbf{R}_{\ell}(t)\right]=\sum_{\mathrm{q} \lambda} \overline{\frac{\hbar}{2 N}} \frac{\hbar}{M_{\ell .} W_{\lambda}}$,

$$
\left\{\left(\mathbf{K}_{\mathrm{r}} \cdot \mathbf{e}_{\mathbf{q}_{\lambda}}^{L}\right)\left(\mathbf{K}_{\mathrm{r}} \cdot \mathbf{e}_{\mathbf{q}^{\lambda}}^{l^{*}}\right) \exp \left[i w_{\lambda}\left(T+t^{\prime}-t\right)\right]-\left(\mathbf{K}_{\mathrm{r}} \cdot \mathbf{e}_{\mathbf{q} \lambda}^{e_{\lambda}}\right)\left(\mathbf{K}_{\mathrm{f}} \cdot \mathbf{e}_{\mathbf{q}}^{l^{*}}\right) \exp \left[-i w_{\lambda}^{\prime}\left(T+t^{\prime}-t\right)\right]_{i}^{\prime}\right.
$$

$\left[-\mathbf{K}_{\mathrm{i}} \cdot \mathbf{R}_{t \cdot}(T), \mathbf{K}_{\mathrm{r}} \cdot \mathbf{R}_{l}\left(T+t^{\prime}\right)\right]=\sum_{\mathbf{q} \lambda} \frac{\hbar}{2 N M_{l}, W_{\lambda}}$,

$$
\left\{\left(\mathbf{K}_{\mathrm{f}} \cdot \mathbf{e}_{\mathbf{q} \lambda}^{L}\right)\left(\mathbf{K}_{\mathrm{i}} \cdot \mathbf{e}_{\mathbf{q} \lambda}^{L^{*}}\right) \exp \left(-i w_{\lambda} t^{\prime}\right)-\left(\mathbf{K}_{\mathrm{f}} \cdot \mathbf{e}_{\mathbf{q} \lambda}^{l^{*}}\right)\left(\mathbf{K}_{\mathrm{i}} \cdot \mathbf{e}_{\mathbf{q}\rangle}^{L}\right) \exp \left(i w_{\lambda} t^{\prime}\right)\right\}
$$

$\left[\mathbf{K}_{i} \cdot \mathbf{R}_{\imath}(T), \mathbf{K}_{\mathrm{r}} \cdot \mathbf{R}_{i}(t)\right]=\sum_{\mathbf{q} ;} \frac{\hbar}{2 N M_{t}, W_{i}}$,

$$
\left\{\left(\mathbf{K}_{\mathrm{i}} \cdot \mathbf{e}_{\mathbf{q} \lambda}^{L}\right)\left(\mathbf{K}_{\mathrm{f}} \cdot \mathbf{e}_{\mathbf{q}_{\lambda} \lambda}^{L^{*}}\right) \exp \left[-i w_{i}(T-t)\right]-\left(\mathbf{K}_{\mathrm{f}} \cdot \mathbf{e}_{\mathbf{q} ;}^{\prime}\right)\left(\mathbf{K}_{\mathrm{i}} \cdot \mathbf{e}_{\mathbf{q} \lambda}^{l_{\lambda}^{*}}\right) \exp \left[i w_{;}(T-t)\right]\right\} .
$$

The thermal average can also be evaluated in a straightforward manner. We are left with:

$$
\begin{aligned}
& -\stackrel{1}{2}\left\langle\left[-\mathbf{K}_{\mathrm{i}} \cdot \mathbf{R}_{l}(T)+\mathbf{K}_{\mathrm{f}} \cdot \mathbf{R}_{L}\left(T+t^{\prime}\right)-\mathbf{K}_{\mathrm{f}} \cdot \mathbf{R}_{L}(t)+\mathbf{K}_{\mathrm{i}} \cdot \mathbf{R}_{l}\right]^{2}\right\rangle_{T}
\end{aligned}
$$

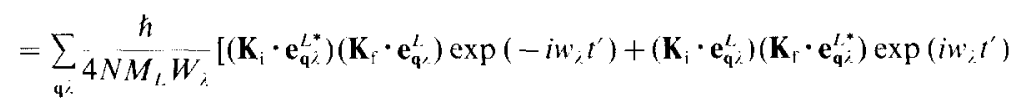

$$
\begin{aligned}
& -\left(\mathbf{K}_{\mathrm{i}} \cdot \mathbf{e}_{\mathbf{q} \lambda}^{l}\right)\left(\mathbf{K}_{\mathrm{f}} \cdot \mathbf{e}_{\mathbf{q}_{\lambda}^{\prime *}}^{l *}\right) \exp \left[-i w_{\lambda}(T-t)\right]-\left(\mathbf{K}_{\mathrm{i}} \cdot \mathbf{e}_{\mathbf{q}}^{L *}\right)\left(\mathbf{K}_{\mathrm{i}} \cdot \mathbf{e}_{\mathbf{q}_{\lambda} \lambda}^{L}\right) \exp \left[i w_{\lambda}(T-t)\right] \\
& -\left(\mathbf{K}_{\mathrm{i}} \cdot \mathbf{e}_{\mathbf{q}}^{l \cdot}\right)\left(\mathbf{K}_{\mathrm{f}} \cdot \mathbf{e}_{\mathbf{q} \lambda}^{L}\right) \exp \left[-i w_{\lambda}\left(T+t^{\prime}\right)\right]-\left(\mathbf{K}_{\mathrm{i}} \cdot \mathbf{e}_{\mathbf{q} \lambda}^{\prime}\right)\left(\mathbf{K}_{\mathrm{f}} \cdot \mathbf{e}_{\mathbf{q} \lambda}^{l^{*}}\right) \exp \left[i w_{\lambda}\left(T+t^{\prime}\right)\right] \\
& +\left(\mathbf{K}_{\mathrm{f}} \cdot \mathbf{e}_{\mathbf{q} \lambda}^{t}\right)^{2} \exp \left[-i w_{\lambda}\left(T+t-t^{\prime}\right)\right]-\left(\mathbf{K}_{\mathrm{f}} \cdot \mathbf{e}_{\mathbf{q},}^{L}\right)^{2} \exp \left[i w \cdot\left(T+t^{\prime}-t\right)\right]
\end{aligned}
$$

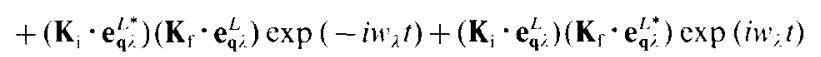

$$
\begin{aligned}
& \left.+\left(\mathbf{K}_{\mathrm{i}} \cdot \mathbf{e}_{\mathbf{q} ;}^{l}\right)^{2} \exp \left(-i w_{i} T\right)+\left(\mathbf{K}_{\mathrm{i}} \cdot \mathbf{e}_{\mathbf{q} \lambda}^{l}\right)^{2} \exp (i w ; T)-2\left(\mathbf{K}_{\mathrm{i}} \cdot \mathbf{e}_{\mathbf{q} ;}^{l}\right)^{2}-2\left(\mathbf{K}_{\mathrm{i}} \cdot \mathbf{e}_{\mathbf{q} ;}^{l}\right)^{2}\right] \\
& \times\left[\left\langle a_{\mathbf{q} i} a_{\mathbf{q} \hat{i}}^{+}\right\rangle_{T}+\left\langle a_{\mathbf{q} i}^{+} a_{\mathbf{q} \lambda}\right\rangle_{T}\right] .
\end{aligned}
$$

The thermal averages $\left\langle a_{\mathbf{q}} ; a_{\mathrm{q}}^{+}\right\rangle_{T}$ and $\left\langle a_{\mathbf{q} ;}^{+} a_{\mathrm{q} ;}\right\rangle_{T}$ are:

$$
\left\langle a_{\mathbf{q} i}^{+} a_{\mathbf{q} \lambda}\right\rangle_{r}=\left[1-\exp \left(-\beta \hbar w_{i}\right)\right] \sum_{n_{\mathbf{q}},} n_{\mathbf{q} i} \exp \left(-\beta \hbar w_{\lambda} n_{\mathbf{q} \lambda}\right)=\frac{1}{\exp \left(\beta \hbar w_{\lambda}\right)-1}
$$

and

$$
\left\langle a_{\mathbf{q} i} a_{\mathbf{q} \lambda}^{+}\right\rangle_{T}=\frac{\exp \left(\beta \hbar w_{j}\right)}{\exp \left(\beta \hbar w_{j}\right)-1} .
$$

The sum of thermal averages yields:

$$
\left\langle a_{\mathbf{q} ;} a_{\mathbf{q} i}^{+}\right\rangle_{T}+\left\langle a_{\mathbf{q} ;}^{+} a_{\mathbf{q} \lambda}\right\rangle_{T}=\operatorname{coth} \frac{\beta \hbar w_{i}}{2} .
$$

We define the following function :

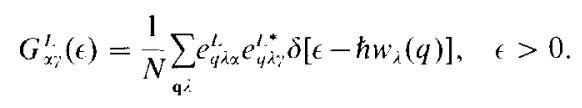

We extend these functions by defining them such that: 


$$
\int_{0}^{\infty} \mathrm{d} \epsilon G_{x_{i}}^{L}(\epsilon)=\delta_{x_{i}} \quad \text { and } G_{x \dot{\delta}}^{L}(\epsilon)=G_{\alpha \bar{\gamma}}^{L}(-\epsilon) .
$$

It follows from equations (55) and (38) that:

$$
\sum_{x} \sum_{L} G_{x \gamma}^{L}(\epsilon)=f(\epsilon)
$$

where $f(\epsilon)$ is the usual phonon frequency distribution function, defined to be $1 / N$ times the number of normal mode frequencies per unit energy interval at $\epsilon$ :

$$
f(\epsilon)=\frac{1}{N} \sum_{\mathbf{q} \lambda} \delta\left[\epsilon-\hbar w_{i}(\mathbf{q})\right] .
$$

The maximum amount of information concerning the dynamics of a crystal which can be extracted from an incoherent neutron scattering experiment (resonant or nonresonant) is the determination of the functions $G_{x_{i}}^{L}(\epsilon)$. The $G$-matrix may be interpreted as an amplitude-weighted directional frequency spectrum.

For a crystal with cubic symmetry, the $G$-matrix is diagonal :

$$
G_{x y}^{L}(\epsilon)=G^{L}(\epsilon) \delta_{x_{i}},
$$

where

$$
G^{L}(\epsilon)=G_{x \alpha}^{L}(\epsilon), \quad \alpha=x, y, z
$$

and if there is one atom per unit cell:

$$
G_{x_{i}}^{L}(\epsilon)={ }_{3}^{1} f(\epsilon) \delta_{x_{i} \cdot}
$$

In terms of $G(\epsilon)$, expression (36) can be written as:

$$
\left\langle\exp \left[-i \mathbf{K}_{\mathrm{i}} \cdot \mathbf{R}_{L .}(T)\right] \exp \left[i \mathbf{K}_{\mathrm{f}} \cdot \mathbf{R}_{L}\left(T+t^{\prime}\right)\right] \exp \left[-i \mathbf{K}_{\mathrm{f}} \cdot \mathbf{R}_{L}(t)\right] \exp \left(i \mathbf{K}_{\mathrm{i}} \cdot \mathbf{R}_{L}\right)\right\rangle_{T}=\exp \left[-\gamma\left(T, t^{\prime}, t\right)\right],
$$

where $\gamma\left(T, t^{\prime}, t\right)$ is defined:

$$
\begin{aligned}
\gamma\left(T, t^{\prime}, t\right)=\int_{0}^{\infty} \frac{\mathrm{d} \epsilon}{\epsilon} \sum_{\alpha} & G_{\alpha \alpha}^{L}(\epsilon)\left\{\operatorname { c o t h } \frac { \beta \epsilon } { 2 } \left[\frac{\hbar^{2} K_{\mathrm{i}}^{2}}{2 M_{L}}\left(1-\cos \frac{\epsilon T}{2}\right)+\frac{\hbar^{2} K_{\mathrm{f}}^{2}}{2 M_{L}}\left(1-\cos \frac{\epsilon\left(T+t^{\prime}-t\right)}{\hbar}\right)\right.\right. \\
+ & \left.\frac{\hbar^{2} \mathbf{K}_{\mathbf{i}} \mathbf{K}_{\mathrm{f}}}{2 M_{L}}\left(\cos \frac{\epsilon\left(T+t^{\prime}\right)}{\hbar}-\cos \frac{\epsilon t^{\prime}}{\hbar}+\cos \frac{\epsilon(T-t)}{\hbar}-\cos \frac{\epsilon t}{\hbar}\right)\right]+i\left[\frac{\hbar^{2} K_{\mathrm{i}}^{2}}{2 M_{L}} \sin \frac{\epsilon T}{\hbar}\right. \\
& \left.\left.+\frac{\hbar^{2} K_{\mathrm{f}}^{2}}{2 M_{t}} \sin \frac{\epsilon\left(T+t^{\prime}-t\right)}{\hbar}+\frac{\hbar^{2} \mathbf{K}_{\mathrm{i}} \mathbf{K}_{\mathrm{f}}}{2 M_{L}}\left(-\sin \frac{\epsilon\left(T+t^{\prime}\right)}{\hbar}-\sin \frac{\epsilon t^{\prime}}{\hbar}-\sin \frac{\epsilon(T-t)}{\hbar}+\sin \frac{\epsilon t}{\hbar}\right)\right]\right\} .
\end{aligned}
$$

For sufficiently short collision times we can expand $\gamma\left(T, t^{\prime}, t\right)$ as :

$$
\exp \left[\gamma\left(T, t^{\prime}, t\right)\right]=\exp \left[-t\left(T+t^{\prime}-t\right) \frac{\hbar \Delta K^{2}}{2 M_{L}}-i\left(t^{\prime}-t\right) \frac{\hbar K_{\mathrm{i}}^{2}}{2 M_{L}}-\frac{\left[\Delta \mathbf{K} T+\mathbf{K}_{\mathrm{f}}\left(t-t^{\prime}\right)\right]^{2}}{2 M_{L} \beta_{\mathrm{cff}}} .\right]
$$

This is the form that Holger (1979) has derived for high temperatures for an ideal gas. However (63) has an effective temperature. Based on this, it seems clear that resonance scattering can be treated using an ideal gas result with the same effective temperatures as for absorption. In addition to this, the differential scattering cross section for the ideal gas was written in terms of the usual $\psi$-function formula so that the effect of chemical binding in the resonance scattering is not large enough to account for the measured effect. $\beta_{\mathrm{eff}}^{-1}$ is the effective temperature in energy units determined by the crystal phonon spectrum. This is given by:

$$
\beta_{\mathrm{efr}}^{-1}=\frac{1}{2} \int_{0}^{\infty} \mathrm{d} \epsilon \epsilon \operatorname{coth}\left(\frac{\epsilon}{2 T_{\mathrm{Re}}} K_{\mathrm{B}}\right) \sum_{\alpha x} G_{\alpha \alpha}^{\prime}(\epsilon)
$$

where $T_{\mathrm{Re}}$ is the real temperature.

The usual expression for incoherent non-resonant scattering (Van Hove, 1954; Turchin, 1965): 


$$
\exp [\gamma(T)]=\exp \left(-i T \frac{\hbar \Delta K^{2}}{2 M_{L}}-\frac{[\Delta \mathbf{K} T]^{2}}{2 M_{t} \beta_{\mathrm{ell}}}\right),
$$

is obtained by setting $t^{\prime}$ and $t$ to be zero. This reduces the collision to a simple potential scattering (Trammel, 1966). Thus the time variables $t$ and $t^{\prime}$ are responsible for the resonance character of the reaction and we are led to believe that $t^{\prime}$ and $t$ can be approximated by zero when $\gamma$ is very large, which the lifetime of the state is very short. This argument reproduces the result of Wick (1954). If $t$ and $t^{\prime}$ are set to be zero in equation (63), the resulting function of $T$ is proportional to the potential scattering cross section where $T$ represents the duration of the interaction for this case.

\section{CONCLUSION}

We have shown that the resonance line shapes are the same for both absorption and scattering for an ideal gas scatterer. We have also shown that the resonant scattering cross section for a harmonic crystal can be approximated by an ideal gas with an effective temperature at high temperatures or large resonance energies. This effective temperature is the same as the one that is used to approximate resonant absorption. There have been a large number of calculations showing that the line shape for resonant absorption in a harmonic crystal is well-characterized by the ideal gas result with an effective temperature and that the chemical binding effect is very small at high temperatures or resonance energies. Thus, we must conclude that, at the temperatures and energies of interest in reactor physics, the chemical binding effect can be ignored for both absorption and scattering. The experiments (Tsang and Brugger, 1979, 1980; Fujita et al., 1984) that show a large chemical binding effect at high energies and temperatures are inconsistent with all of the theoretical results and should be reevaluated.

\section{REFERENCES}

Bernabei A. (1964) Report BNL-860-T-344.

Bethe H. and Placzek G. (1937) Phys. Rer. 51, 450.

Bloch F. (1932) Z. Phys. 74, 295.

Born M. and Huang K. (1954) Dynamical Theory of Crystal Lattice. Oxford University Press, New York.

Djafri D. (1988) Ann. nucl. Energy 15, 75.

Fujita Y., Brugger R., Alger D. and Miller W. (1984) J. Nucl. Sci. Technol. 21, 83.

Goodman B. and Waller I. (1966) Arkiv. For. Fysik. 32, 537.

Hass R. and Shore F. J. (1959) Rev. Sci. Instrum. 30, 17.

Holger S.-J. (1979) Ph.D. Thesis, Georgia Institute of Technology.

Holger S.-J. and Karam R. A. (1981) Ann. nucl. Energy 8, 255.

Karam R. A. (1975) Int. Conf. on $U^{235}$ Cross Sections, Paper BN-NCS 50541.

Koppel J. U. and Houston D. H. (1968) Report GA-8774, U.S. Atomic Energ. Commission.

Lamb W. E. (1939) Phys. Rev. 55, 190.

Mössbauer R. L. (1958) Z. Physik 124, 15 I.

Nelkin M. and Parks D. (1960) Phys. Rev. 119, 1060.

Osborn R. K. and Yip S. (1966) The Foundations of Neutron Transport Theory. Gordon \& Breach, New York.

Shamaoun A. (1989) Ph.D. Thesis, The University of Michigan.

Shamaoun A. and Summerfield G. (1989) Ann. nucl. Energy 16, 263.

Sher R. (1983) Report ZPR-TM-448, Argonne National Laboratory.

Singwi K. S. and Sjolander A. (1960) Phys. Rev. 120, 1093.

Summerficld G. C. (1965) J. Chem. Phys. 43, 1079.

Trammel G. T. (1966) Phis. Ret. 141, 815.

Tsang F. and Brugger R. (1979) Nucl. Sci. Engng 72, 52.

Tsang F. and Brugger R. (1980) Nucl. Sci. Engng 74, 34.

Turchin V. IF. (1965) Slow Neutron Scattering. Daniel Dakey, New York.

Van Hove L. (1954) Phys. Rev. 95, 249.

Weinreich G. (1965) Solids: Elementary Theory for Advanced Students. Wiley, New York.

Westinghouse Atomic Power Division (1954) Report SR-506.

Weyl H. (1927) Z. Phys. 46, 1.

Wick G. (1954) Phys. Rev. 94, 1228.

Word R. E. and Trammell G. T. (1981) Phys. Ret. B24, 2430. 